\title{
Perspectivas e desafios do núcleo de apoio à saúde da família quanto às práticas em saúde
}

Prospects and challenges of core support for family health as to practice in health

Karla Ferraz dos Anjos', Saulo Sacramento Meira², Carla Eloá de Oliveira Ferraz³, Alba Benemérita Alves Vilela ${ }^{4}$, Rita Narriman Silva de Oliveira Boery ${ }^{5}$ Edite Lago da Silva Sena ${ }^{6}$

\author{
'Mestranda do Programa de Pós- \\ Graduação em Enfermagem e Saúde \\ da Universidade Estadual do Sudoeste \\ da Bahia. \\ karla.ferraz@hotmail.com \\ ${ }^{2}$ Mestrando do Programa de Pós- \\ Graduação em Enfermagem e Saúde \\ da Universidade Estadual do Sudoeste \\ da Bahia. \\ saulo_meira@hotmail.com \\ ${ }^{3}$ Mestranda do Programa de Pós- \\ Graduação em Enfermagem e Saúde \\ da Universidade Estadual do Sudoeste \\ da Bahia. \\ caueloa@yahoo.com.br \\ ${ }^{4}$ Doutora em Enfermagem pela \\ Universidade Federal do Ceará (UFC) \\ - Fortaleza (CE), Brasil. Professora \\ do Programa de Pós-Graduação em \\ Enfermagem e Saúde da Universidade \\ Estadual do Sudoeste da Bahia (UESB) - \\ Jequié (BA), Brasil. \\ albavilela@gmail.com \\ ${ }^{5}$ Doutora em Enfermagem pela \\ Universidade de São Paulo (USP) - São \\ Paulo (SP), Brasil. Professora do Programa \\ de Pós-Graduação em Enfermagem \\ e Saúde da Universidade Estadual do \\ Sudoeste da Bahia Universidade Estadual \\ do Sudoeste da Bahia (UESB) - Jequié \\ (BA), Brasil. \\ rboery@gmail.com
}

${ }^{6}$ Doutora em Enfermagem pela Universidade Federal de Santa Catarina (UFSC) - Florianópolis (SC), Brasil. Professora do Programa de PósGraduação em Enfermagem e Saúde da Universidade Estadual do Sudoeste da Bahia (UESB) - Jequié (BA), Brasil. editelago@gmail.com
RESUMO: O Núcleo de Apoio à Saúde da Família (NASF) surgiu como proposta para meIhorar as práticas de saúde. Entretanto, desde sua criação, inúmeros desafios surgiram, configurando uma problemática que necessita ser modificada. Assim, o objetivo é refletir sobre as perspectivas e os desafios do NASF quanto às práticas em saúde. Trata-se de uma revisão de literatura realizada por meio de consulta às bases de dados da SciELO e Lilacs no período de abril a maio de 2012. Concluiu-se que, apesar de o NASF ser reconhecido como suporte à Estratégia Saúde da Família (ESF), ainda não atua de forma articulada, sendo imprescindível que ocorram mudanças na organização dos serviços e na conduta dos profissionais de saúde.

PALAVRAS-CHAVE: Atenção Primária à Saúde; Equipe de assistência ao paciente; Políticas públicas de saúde.

ABSTRACT: The Support Center for Family Health (NASF) has emerged as a proposal to improve health practices. However, since its inception, many challenges have surfaced, setting up a problem that needs to be modified. Thus, the objective of this article is to think over NASF' prospects and challenges as for health practices. This is a literature review carried out by surveying SciELO and Lilacs databases within the period April-May 2012. It was concluded that, yet being recognized as a support to the Family Health Strategy (FHS), NASF does not act articulately, making changes imperative in the organization of services and in the conduct of health professionals.

KEYWORDS: Primary Health Care; Patient care team; Public health policy. 


\section{Introdução}

O Núcleo de Apoio à Saúde da Família (NASF) surgiu como proposta tanto para prestar assistência às demandas populacionais não alcançadas pelas equipes que compóem a Estratégia de Saúde da Família (ESF) como para apoiar essas equipes na efetivação da rede de serviços e ampliar sua abrangência, visando melhorar a qualidade da assistência à população no nível da Atenção Básica, que carece de açóes mais eficazes.

Em contraste com os modelos convencionais de prestação de cuidados, que primam pela assistência curativa, especializada, fragmentada e individual, a proposta de trabalho do NASF é a de superar essa lógica em direção à corresponsabilização e à gestão integrada do cuidado por meio de atendimentos compartilhados e projetos terapêuticos que envolvam os usuários e sejam capazes de considerar a singularidade dos sujeitos assistidos.

A implantação do NASF representa a busca crescente pela integralidade da atenção e interdisciplinaridade nas açôes em saúde, consoante a perspectiva de consolidação da ESF. Nesse sentido, o NASF é ambicioso e pode até parecer utópico, principalmente quando se avalia a formação dos profissionais de saúde, que, em grande parte, ainda encontram-se distantes das perspectivas com as quais o NASF foi criado (MÂNGIA; LANCMAN, 2008).

Refletindo-se sobre a trajetória do NASF desde a sua criação até os dias atuais, observa-se que as expectativas com a criação desse núcleo foram pautadas no alargamento da oferta de serviços de saúde na ESF, tanto em aspectos quantitativos como qualitativos. Entretanto, essa realidade ainda não se concretizou, e isso se deve, principalmente, ao déficit de formação e capacitação dos profissionais de saúde que o compóem, bem como daqueles que integram as equipes da ESF, haja vista que vários desses profissionais também possuem uma visão fragmentada em relação ao cuidado, implicando diretamente na qualidade da assistência prestada, seja ela individual ou coletiva.

O NASF é constituído por uma equipe de apoio e o objetivo principal de suas ações é possibilitar maior qualidade à assistência prestada e não apenas "suprir a demanda”. Apresenta, dentre seus desafios, a necessidade de mudança da cultura organizacional no Sistema Único de Saúde (SUS), que, historicamente, vem priorizando a quantidade de procedimentos em detrimento de sua qualidade; o referenciamento em prejuízo da resolubilidade na Atenção Básica; e a avaliação de impacto e indicadores de saúde com abordagem simplesmente quantitativa (NASCIMENTO; OLIVEIRA, 2010).

Considerando-se a assistência à saúde oferecida pelo SUS e a perspectiva que se tem acerca do NASF, tornam-se necessárias mudanças nesse sistema, sejam elas no âmbito da gestão ou do domínio da execução de açóes qualificadas dos profissionais de saúde, uma vez que a cultura predominante nas práticas em saúde ainda é considerada um desafio que o NASF precisa superar para conseguir validar, de fato, suas diretrizes.

Este estudo teve como objetivo refletir sobre as perspectivas e os desafios do NASF quanto às práticas em saúde, tomando como base a literatura existente sobre implantação, desafios e conquistas do NASF.

\section{Método}

Trata-se de revisão de literatura inicialmente realizada por meio da Biblioteca Virtual de Saúde (BVS), que abriga bases de dados reconhecidas. A busca dos estudos ocorreu no período de abril a maio de 2012, a partir dos seguintes descritores: "Atenção Primária à Saúde", "Equipe de Assistência ao Paciente" e "Políticas Públicas de Saúde". Na articulação das palavras, foi adotada a expressão booleana 'AND', que permite a inserção de duas ou mais palavras. $\mathrm{O}$ resultado conduziu a pesquisa nas bases de dados Scientific Electronic Library Online (SciELO) e Literatura Latino-Americana e do Caribe em Ciências (Lilacs). A pesquisa estendeu-se aos arquivos do Ministério da Saúde (Brasil).

Os critérios utilizados para inclusão do material acessado ao estudo foram: disponibilidade on line do texto completo, período de publicação entre 2007 e 2012 e idioma português. Foram encontrados 36 artigos relacionados ao tema, dentre os quais seis foram selecionados por serem pertinentes ao objeto do estudo e se enquadrarem nos critérios de inclusão estabelecidos. 
Os artigos selecionados foram analisados com base no objetivo proposto e na relevância científica e social. Em seguida, realizou-se a leitura dos arquivos por todos os autores, em momentos distintos. Posteriormente, em conjunto, foram distribuídos os arquivos nos quatro eixos temáticos, a saber: histórico e caracterização do NASF; o NASF e a ESF; o NASF na prática; e enfrentamentos do NASF.

\section{Resultados e discussão}

A ESF, como estratégia pública articuladora de cuidados primários à saúde do indivíduo, da família e da comunidade, deveria percorrer caminhos e, assim, modificar-se levando em conta os determinantes sociais da saúde de determinada localidade. De forma concomitante, o NASF tem aporte legal e instrumental passível de aproximação com essa realidade, desde que se configure num apoio matricial efetivo para as ESF (ANDRADE et al., 2012).

\section{HISTÓRICO E CARACTERIZAÇÃO DO NASF}

O Ministério da Saúde (MS) criou os NASF 1 e 2 por meio da Portaria GM/MS no 154 , publicada em 4 de março de 2008 com o objetivo de ampliar a abrangência das açôes da Atenção Básica (AB), bem como maximizar sua resolubilidade, apoiando a inclusão da ESF na rede de serviços e nos processos de territorialização e regionalização a partir da Atenção Básica (BRASIL, 2008).

O NASF, em 2008, era dividido em três modalidades: NASF 1, NASF 2 e NASF 3. O NASF 1 foi desenvolvido para atender os municípios com contingente populacional acima de 20.000 habitantes. Entretanto, como cerca de $80 \%$ dos municípios brasileiros contam com uma população menor que 20.000 habitantes, e vários deles necessitam mais de acompanhamento à saúde do que os municípios mais populosos, o Governo Federal, visando a ampliar a cobertura da ação, criou o NASF 2, destinado a acolher os municípios com densidade demográfica de até 10 habitantes por $\mathrm{km}^{2}$ (BRASIL, 2010a).
No âmbito do SUS, a modalidade NASF 3 foi criada a partir da Portaria GM/MS no 2.843, de 20 de setembro de 2010 , e tinha como prioridades a atenção integral em saúde e a saúde mental - assistindo, principalmente, usuários de crack, álcool e outras drogas, na Atenção Básica - em municípios com população inferior a 20.000 habitantes (BRASIL, 2010a).

Com a perspectiva de suprir lacunas que, inicialmente, não haviam sido previstas no campo da Atençáo Básica e que deveriam ser priorizadas - principalmente por se tratar de grupos populacionais -, a inserção da saúde mental e da reabilitação, como parte da integração à rede de serviços, tornou-se necessária, como aconteceu a partir da implantação e implementação do NASF 3. Assim, fortaleceu-se a interdisciplinaridade, a intersetorialidade, o território, a educação permanente em saúde, a promoção da saúde e a humanização (MÂNGIA; LANCMAN, 2008).

As Portarias GM/MS no 154/2008 e 2.843/2010 foram revogadas pela Portaria GM/MS no 2.488 , de 2 de outubro de 2011. Nesse contexto, ocorreram mudanças no NASF, que passou a ser organizado em apenas duas modalidades: NASF 1 e NASF 2 (BRASIL, 2011a). Ressalte-se que os dispositivos das portarias anteriores que não conflitam com a atual portaria permanecem em vigor.

O NASF 3 foi suprimido a partir da publicação da Portaria GM/MS no 2.488/2011 e se tornou automaticamente NASF 2. Os municípios com projetos de NASF 3 anteriormente enviados ao MS deverão, atualmente, enviar para a Comissão Intergestores Bipartite documento que informe as alterações ocorridas. Fica garantido o financiamento dos NASF intermunicipais já habilitados em data anterior, mas fica extinta a possibilidade de implantação de novos NASF intermunicipais (BRASIL, 2011a).

A composição do NASF baseia-se na proposta de equipe de referência, ou seja, toma por base aqueles que têm a responsabilidade pela condução de um caso individual, familiar ou comunitário, objetivando ampliar as possibilidades de construção do vínculo entre profissionais e usuários. Esse tipo de arranjo busca mudar o padrão dominante das responsabilidades nas organizaçóes e construir a responsabilidade de pessoas por pessoas. 
Conforme alteraçóes apresentadas na Portaria GM/MS no 2.843, publicada em 20 de setembro de 2010, o NASF 1 realiza suas atividades vinculado a, no mínimo, oito e, no máximo, quinze Equipes de Saúde da Família ou equipes de atençâo básica para populações específicas. A exceção é para municípios com menos de 100.000 habitantes dos Estados da Amazônia Legal e Pantanal Sul-mato-grossense, onde cada NASF 1 poderá realizar suas atividades vinculado a, no mínimo, cinco e, no máximo, nove equipes. Quanto ao NASF 2, deve realizar suas atividades vinculado a, no mínimo, três e, no máximo, sete Equipes de Saúde da Família (BRASIL, 2011a).

Os NASF 1 e 2 deverão ter uma equipe formada por profissionais de nível superior, a ser definida pelos gestores municipais a partir dos dados epidemiológicos, das necessidades locais e das equipes de saúde que apoiarão. Assim, os profissionais que os compóem são: Médico Acupunturista, Assistente Social, Profissional de Educação Física, Fisioterapeuta, Fonoaudiólogo, Farmacêutico, Médico Ginecologista, Obstetra, Médico Homeopata, Nutricionista, Médico Pediatra, Psicólogo, Terapeuta Ocupacional, Médico Psiquiatra, Médico Geriatra, Médico Internista, Médico do Trabalho, Médico Veterinário, profissional com formação em arte e educação e profissional de saúde sanitarista (BRASIL, 2011a). É relevante observar que não há diferença entre os NASF quanto à composição dos profissionais.

O NASF trabalha com a equipe de referência, que é definida também por uma coordenação, gerência, comum. É dever de tal equipe desconstruir, nas organizaçóes de saúde, o modelo de 'linhas de produção' fragmentadas, nas quais o poder gerencial se atrela ao saber disciplinar fragmentado e as chefias se dividem por corporaçóes, produzindo arranjos que desvalorizam ou rivalizam as categorias envolvidas no processo de atenção à saúde.

A organização e o desenvolvimento do processo de trabalho dos NASF dependem de algumas estratégias já testadas no Brasil, como é o caso do Apoio Matricial, da Clínica Ampliada, do Projeto Terapêutico Singular (PTS) e do Projeto de Saúde no Território (PST) (MARTINES; CHAVES, 2007).
Em definiçãao aos modelos fundamentais de funcionamento do NASF, deve-se considerar o Apoio Matricial, que apresenta as dimensóes do suporte assistencial e técnico-pedagógico. A dimensão assistencial é aquela que vai produzir açâo clínica direta junto aos usuários. Já a dimensão técnico-pedagógica vai produzir ação de apoio educativo com e para a equipe. Essas duas dimensóes podem e devem ser associadas em diversos momentos, uma vez que não é possível a nenhum trabalhador em saúde esquivar-se de lidar, em alguma medida, com os afetos nas relaçóes terapêuticas (BARBOSA et al., 2012).

A proposta de Clínica Ampliada direciona-se a todos os profissionais que fazem clínica, em um recorteum destaque de sintomas e informaçóes - de sua competência profissional. Já o PTS, constitui um conjunto de condutas terapêuticas articuladas para cada sujeito individual ou coletivo, resultado da discussão coletiva de uma equipe interdisciplinar e, se necessário, com apoio matricial (FIGUEIREDO, 2012).

Também utiliza como ferramenta de trabalho das Equipes de Saúde da Família e do NASF a proposta do PST com o intuito de desenvolver açóes efetivas na produçâo da saúde em um território, articulando os serviços de saúde com outros serviços e políticas sociais, de forma a investir na qualidade de vida e na autonomia das comunidades (FIGUEIREDO, 2012).

\section{NOVOS CENÁRIOS E PROJETOS QUE AMPLIAM A ATUAÇÃO DO NASF}

Conforme o Decreto Federal no 7.508/2011, que regulamenta a Lei no 8.080/1990 e dispóe sobre a organização do sistema público de saúde, planejamento da saúde, assistência à saúde e articulação interfederativa, é possível pensar na reestruturação do NASF por meio da implantação das "Regiốes de Saúde". Uma Região de Saúde é um espaço geográfico contínuo constituído por agrupamento de municípios limítrofes, delimitado a partir de identidades culturais, econômicas e sociais, e infraestrutura de transportes e redes de comunicação compartilhadas, com a finalidade de integrar a organização, o planejamento e a execução de açôes e serviços de saúde (BRASIL, 2011c). 
Aparece, aqui, uma dificuldade frequentemente enfrentada pelos municípios que possuem menos de 20.000 habitantes e que, até então, não poderiam sediar o NASF 1. Cada região deve oferecer serviços de atenção primária, urgência e emergência, atenção psicossocial, atenção ambulatorial especializada e hospitalar e, por fim, vigilância em saúde (BRASIL, 2011b).

Os NASF, compostos por profissionais de diferentes áreas de conhecimento, devem atuar de maneira integrada e apoiando os profissionais das Equipes de Saúde da Família e das Equipes de Atençâo Básica para populaçóes específicas. Assim, foram incorporadas como novas modalidades de equipes de $\mathrm{AB}$ algumas que já existiam, tais como os Consultórios de Rua, além das Equipes de Saúde da Família ribeirinhas e unidades móveis fluviais, com desenho e financiamento específicos e mais adequados às realidades em que atuam. Com isso, a ação dos profissionais dos NASF irá colaborar no sucesso do programa Academia da Saúde, criado pela Portaria GM/MS no 719/2011 (BRASIL, 2011b).

Nos Consultórios de Rua, as equipes deverão realizar suas atividades de forma itinerante, desenvolvendo ações na rua, em instalações específicas, na unidade móvel e nas instalações de Unidades Básicas de Saúde do território onde estão atuando, sempre articuladas e desenvolvendo açóes em parceria com as demais Equipes de Atenção Básica do território - as UBS e os NASF -, com os Centros de Atenção Psicossocial, a Rede de Urgência e os serviços e instituiçóes componentes do Sistema Único de Assistência Social, dentre outras instituiçôes públicas e da sociedade civil (BRASIL, 2011a).

As Equipes de Saúde da Família para o atendimento da População Ribeirinha podem desempenhar a maior parte de suas funçóes em unidades básicas de saúde localizadas nas comunidades pertencentes à área adscrita. Já as Equipes de Saúde da Família Fluviais, desempenham suas funçôes em Unidades Básicas de Saúde Fluviais, a fim de prestarem assistência àquela população (BRASIL, 2011a). Nesse sentido, essas equipes podem ampliar suas intervençóes contando com o apoio dos NASF quando estiverem funcionando no município.

Os NASF podem utilizar como espaço as Academias da Saúde com o objetivo de ampliar a capacidade de intervenção coletiva das equipes de atenção básica para as açóes de promoção de saúde, no sentido de fortalecer o protagonismo de grupos sociais em condiçóes de vulnerabilidade com vistas à superação de sua condição (BRASIL, 2011a).

\section{O NASF E A ESF}

A proposta de criação do NASF constitui importante estratégia para promover o fortalecimento da ESF e, de modo especial, desenvolver e aprimorar um novo modelo de atenção à saúde, voltado ao trabalho de equipe multiprofissional. Diretrizes, como integralidade, qualidade da assistência, equidade e participação social precisam se afirmar em açôes coletivas centradas no desenvolvimento do ser humano e na promoção da saúde, e ser capazes de produzir saúde além do contexto individual, assistencial e medicalizante (MÂNGIA; LANCMAN, 2008).

A ESF propóe uma nova dinâmica para a estruturação dos serviços de saúde, assim como para a relação com a comunidade, envolvendo os diversos níveis de assistência. Assume o compromisso de prestar assistência integral à população, na unidade de saúde e no domicílio, de acordo com suas necessidades, identificando fatores de risco aos quais a população está exposta. Dessa forma, permite à ESF intervir de forma apropriada por meio da humanização das ações em saúde, buscando a satisfação dos usuários via estreito relacionamento dos profissionais com a comunidade e considerando sempre a saúde como um direito da cidadania (ARAÚJO, 2012).

Em paralelo, o NASF propóe-se a estabelecer outro modelo que vise a superar a lógica fragmentada da saúde por meio da construçáo de redes articuladas de atenção e cuidados, funcionando sob as diretrizes de ação interdisciplinar e intersetorial. $\mathrm{O}$ apoio matricial deve atuar de forma corresponsabilizada, reorientando e reorganizando o modelo de gestáo e de atenção com a equipe da ESF.

Vale lembrar que a ESF é um programa brasileiro e vertente da Atenção Primária à Saúde (APS) implantada em 1994, e caracteriza-se como porta de entrada prioritária de um sistema de saúde constituído no direito à saúde e na igualdade do cuidado (BRASIL, 2011c). 
Tratando-se da atenção à saúde voltada à atuação do NASF, suas diretrizes precisam ser levadas em consideração com o intuito de se promover saúde com qualidade e humanização da assistência. Para isso, o NASF precisa se organizar em áreas estratégicas, tais como: atividade física ou práticas corporais; práticas integrativas e complementares; reabilitação; assistência farmacêutica; alimentação e nutrição; serviço social; saúde mental; saúde da mulher; saúde da criança, do adolescente e do jovem (MÂNGIA; LANCMAN, 2008).

O NASF apresenta, em normatizaçóes, as dimensões do suporte assistencial e técnico-pedagógico. Entretanto, intervenções diretas do NASF frente a usuários e famílias só podem ser realizadas sob encaminhamento das ESF com discussóes e negociação, a priori, entre os profissionais responsáveis pelo caso (CAMPOS, 2007; BARBOSA et al., 2007). Em verdade, o atendimento direto e individualizado pelo Apoio Matricial ocorrerá somente em situaçóes extremamente necessárias (BRASIL, 2011c).

A organização dos processos de trabalho do NASF e da ESF deverá criar espaços de discussão voltados à gestão e à constituição de uma rede de cuidados. As reunióes e os atendimentos precisam constituir um processo de aprendizado coletivo, em uma perspectiva de se produzir saúde e de se ampliar a autonomia das pessoas. Além disso, ao se realizar a avaliação diagnóstica, é necessário considerar não somente o saber clínico como também o contexto subjetivo do indivíduo; é fundamental, também, definir a intervenção terapêutica, considerando a complexidade biopsicossocial das demandas de saúde que estão sendo referenciadas (BRASIL, 2010b).

\section{O NASF NA PRÁTICA}

Por se tratar de uma estratégia inovadora, a implantação do NASF implica a necessidade de a equipe criar espaços físicos rotineiros com dimensôes de suporte para realizar reuniốes, planejamentos e discussão de casos, objetivando definir projetos terapêuticos a serem compartilhados por toda a equipe de forma validada e reconhecida por gestores, na forma de PTS e de PST (NASCIMENTO; OLIVEIRA, 2010).
A inserção do NASF deveria modificar a interação dos envolvidos no processo, sendo de fundamental relevância um novo planejamento e o exercício da educação permanente que possibilite sua implantação, adaptando-se o saber-fazer à nova perspectiva implantada. O NASF apresenta potencialidades e fragilidades, sendo necessários ajustes para que a prestação de serviço seja mais eficaz e eficiente (ANDRADE et al., 2012).

A efetividade e a qualidade do acolhimento e acompanhamento da saúde no setor primário devem ocorrer de forma transparente e ser alcançadas através da parceria entre o NASF e a ESF. Isso não acontece de forma espontânea e natural, havendo a necessidade de os profissionais assumirem suas responsabilidades em regime de cogestão entre as equipes e sob a coordenação do gestor local, em processos de constante construção.

Diante das responsabilidades que lhes são confiadas, as equipes do NASF assumem o compromisso com a população e com a ESF, propondo-se a identificar as necessidades de saúde comunitária ao mesmo tempo em que fortalecem as equipes de referência. Seu desempenho deverá ser avaliado não só por indicadores de resultado para a população, mas também por indicadores do resultado da sua ação na equipe (BRASIL, 2011c).

No que se refere ao processo de trabalho dos profissionais, espera-se que este seja estabelecido e pactuado entre gestor, equipe do NASF e ESF; as estratégias devem ser definidas e detalhadas criteriosamente, em função de o NASF ser um setor partilhado por peculiaridades pessoais e profissionais. É inegável, também, a necessidade de se desarticularem antigos conceitos e de se instituírem novas concepçôes e valores coletivos.

Náo obstante os avanços e conquistas da ESF, o NASF representa um marco importante na ampliação das possibilidades de se alcançarem melhores resultados em saúde, com enfoque na promoção da saúde e no cuidado à população, principalmente devido ao apoio e suporte que oferece à ESF. A inclusão de novos profissionais de saúde amplia a possibilidade de se responder aos novos e antigos desafios da morbidade dos brasileiros, tais como o sofrimento psíquico, as modificações no padrão nutricional e o aumento da longevidade da população, que, para o sistema de saúde do Brasil, representa um maior número de pessoas com 
morbidades e, principalmente, com doenças crônicas não degenerativas.

Além disso, essa estratégia traz possibilidades de se aumentar a oferta de práticas integrativas e complementares, além da oferta da melhor tecnologia disponível para parte das doenças crônicas; não obstante, possibilita uma reflexão acerca de tratamentos baseados apenas na medicalização de pacientes (MENDONÇA, 2009).

\section{ENFRENTAMENTOS DO NASF}

Frente à proposta metodológica do NASF no que se refere ao desenvolvimento do trabalho de maneira interdisciplinar, é necessário haver revisão crítica dos processos educativos e formativos que vêm sendo disponibilizados por Instituiçóes de Ensino Superior na formação de profissionais da área da saúde.

Assim, as competências apresentam-se com uma nova perspectiva na formação, embora vários profissionais se deparem com desafios ao trabalhar de maneira inter e transdisciplinar. De forma a corresponder a essas exigências, faz-se necessário refletir sobre a formação e o perfil de competência desejados nessa área, com enfoque não apenas no conhecimento técnico especializado, mas também nas habilidades e atitudes a serem desenvolvidas em prol da saúde da população, de uma forma socialmente responsável (NASCIMENTO; OLIVEIRA, 2010).

Diversos desafios são impostos, principalmente quando se avalia a formação de parte dos profissionais de saúde que atuam nessa estratégia, como a necessidade de mudança na organização dos serviços. Nas práticas de saúde, os profissionais que atuam no NASF apresentam dificuldades em criar possibilidades para a atuação conjunta, integrada e intersetorial que incorpore a participação de usuários na atual concepção ampliada de saúde que está sendo assumida pelo SUS; há dificuldades em aprender, no cotidiano, com a assistência e com o trabalho coletivo no território envolvido, o que depende de flexibilidade e interlocução dos envolvidos (MÂNGIA; LANCMAN, 2008).

Dentre as dificuldades que estão sendo identificadas no processo de trabalho do NASF, destaca-se a formação dos profissionais, que, em parte, não atende às necessidades do SUS e menos ainda da Atenção Básica. A transformação da formação e das práticas é um desafio a ser superado, pois implica mudança de paradigmas já estruturados nos serviços, nas instituiçóes de ensino e nas relações interpessoais. Com o diálogo e a aproximação, no âmbito das práticas e concepçóes atuais de atenção à saúde, poder-se-á diminuir o descompasso entre formação e realidade dos serviços. Assim, favorecer-se-á a construção de uma nova forma de trabalho em saúde centrada no usuário, com qualidade, resolubilidade e equidade (NASCIMENTO; OLIVEIRA, 2010).

Apesar das conquistas e avanços na área da saúde, a implantação do NASF propõe repensar a formação e as práticas em saúde vivenciadas até o momento pela ESF. O NASF traz como ferramentas para o desenvolvimento de seu trabalho a clínica ampliada, o matriciamento, o projeto terapêutico singular e o projeto de saúde com foco no território; tudo isso tendo em vista a realização do cuidado ao usuário, além da qualificação das ações das equipes (NASCIMENTO; OLIVEIRA, 2010).

Por tratar-se de processo em construçáo, a implantação do NASF sugere a necessidade de se estabelecer efetivamente uma qualificação profissional, que só será alcançada por meio de reflexão e diálogo, em torno de uma perspectiva que deve ocorrer tanto na esfera interna de cada profissáo como no campo da saúde como um todo. Com esse perfil profissional, é possível estabelecerem-se estratégias como o planejamento desenhado coletivamente, reconstruindo o sentido e o significado do seu agir. É necessário que sejam inseridas em sua prática por meio da definição de projetos terapêuticos compartilhados por toda a equipe, de forma validada, permitindo açóes significativamente reconhecidas sob o ponto de vista dos gestores (BARBOSA et al., 2010).

A atuação dos profissionais de saúde no NASF depara-se com diversos enfrentamentos, dentre os quais a maneira como as organizaçóes vêm se estruturando, uma vez que 'conspiram' contra o modo interdisciplinar e de interlocução. E, para superar esses desafios, além de trabalhar com a equipe multiprofissional e de maneira interdisciplinar, o sistema precisa de uma cogestão para que os empecilhos sejam conhecidos, analisados e, quando possível, removidos ou enfraquecidos (CAMPOS; DOMITTI, 2007). 
O NASF, desde sua implantação, emana desafios, especialmente no que refere à dificuldade de contemplar a integralidade da saúde individual e coletiva, conforme proposto pelo MS. Por isso, apresenta limitaçóes no alcance de seus serviços.

O MS propóe que a implantação do NASF e sua estratégia de trabalho ocorram de forma interdisciplinar, em que diferentes conhecimentos, saberes, práticas, valores e modos de relacionamento se encontrem para atingir um objetivo comum. São exigidas dos profissionais atitudes de permeabilidade frente aos diferentes saberes e de flexibilidade frente às diversas necessidades, aspectos que constituem um desafio para com a prática, mas que podem ser superados com a experimentação, isto é, com a vivência durante a ação de apoiar os envolvidos (BRASIL, 2010c).

Vários profissionais de saúde supervalorizam a autonomia, considerando-a direito de deliberar sobre casos de modo isolado e definitivo. Por outro lado, o apoio matricial promove encontros entre distintas perspectivas, obrigando os profissionais a comporem projetos terapêuticos com outras racionalidades e visóes de mundo. Entretanto, em casos de impasse - principalmente os de caráter terapêutico -, não há instâncias superiores para resolver o problema, havendo necessidade de o gerente em saúde ter, ao menos, uma pequena capacidade de interferir na conduta específica do especialista. Cabe aos envolvidos no conflito, então, encontrar estratégias que não prejudiquem o usuário nem interfiram no projeto terapêutico do paciente (CAMPOS; DOMITTI, 2007).

\section{Considerações finais}

No sentido de fortalecer o NASF e de ampliar cada vez mais suas ações, estão sendo estimuladas práticas diversas, individuais, coletivas e, principalmente, multidisciplinares, no âmbito das diversas áreas da saúde coletiva na Atenção Básica em Saúde.

Algumas rotinas precisam ser modificadas, e, como consequência, desconfortos serão inevitáveis, instituindo-se consequências diretas à defesa e ao fortalecimento do NASF. Entre as medidas necessárias para a mudança, destacam-se: a reflexão sobre o cotidiano com os atores envolvidos, o investimento no sentido de fortalecer os vínculos e a criação de um espaço para o debate coletivo entre as disciplinas envolvidas no processo de trabalho e na produção do cuidado.

Torna-se indispensável, portanto, a ponderação junto a gestores e organizaçóes comprometidas com a participação social sobre a relação entre a composição das equipes e as necessidades de saúde das comunidades. Importa reconhecer que a qualidade em saúde não depende apenas de uma forma ou modelo, mas também do domínio das competências necessárias ao respectivo exercício profissional.

\section{Referências}

ANDRADE, L.M.B. et al. Análise da implantação dos Núcleos de Apoio à Saúde da Família no interior de Santa Catarina. Saúde e Transformação Social, v.1, n.1, p.18-31, 2012.

ARAÚJO, M.F.S. O enfermeiro no Programa de Saúde da Família: prática profissional e construção da identidade. Conceitos. Disponível em: <http://www.saude.ms.gov.br/controle/ShowFile. php?id=53553>. Acesso em: 26 abr. de 2012

BARBOSA, E.G. et al. Experiência da Fisioterapia no Núcleo de Apoio à Saúde da Família em Governador Valadares - MG. Revista Fisioterapia e Movimento. Disponível em: <http://www.scielo.br/
scielo.php?script=sci_arttext\&pid=S010351502010000200015\&l ng=en>. Acesso em: 27 abr. 2012

BRASIL. Ministério da Saúde. Gabinete do Ministro. Portaria MS/ GM no 2.488, de 21 de outubro de 2011. Brasília: Diário Oficial da União, Poder Executivo, Brasília, DF, Seção 1, p.48-55, 24 out. 2011. 2011a. Disponível em: <http://www.corengo.org.br/attachments/ article/374/PORTARIA\%20MS_GM\%20N\%C2\%BA\%202.488,\%20 DE\%2021\%20DE\%200UTUBRO\%20DE\%202011.pdf>. Acesso em: 30 jan. 2013

Ministério da Saúde. Departamento de Atenção Básica. 
Secretaria de Atenção à Saúde. Saúde mais perto de você: a renovação na saúde. Revista Brasileira Saúde da Família, Ministério da Saúde, Ano XII, n 30. Brasília, DF: Ministério da Saúde, 2011 b. Disponível em: <http://189.28.128.100/dab/docs/publicacoes/ revistas/revista_saude_familia30.pdf>. Acesso em: 30 jan. 2012.

Casa Civil. Subchefia para Assuntos Jurídicos. Decreto no 7.508, de 28 de junho de 2011. 2011c. Disponível em: <http://www. planalto.gov.br/ccivil_03/_ato2011-2014/2011/decreto/D7508. htm>. Acesso em: 31 jan. 2013.

Ministério do Estado da Saúde. Gabinete do Ministro. Portaria no 2.843, de 20 de setembro de 2010. Legislações. Gabinete do Ministro. 2010a. Disponível em: <http://www.brasilsus.com.br/ legislacoes/gm/105513-2843.html>. Acesso em: 22 abr. 2012.

Ministério da Saúde. Secretaria de Atenção à Saúde. Política Nacional de Humanização. Atenção Básica. Caderno Humaniza SUS. 2010b. Disponível em: <http://bvsms.saude.gov. br/bvs/publicacoes/cadernos_humanizasus_atencao_basica. pdf>. Acesso em: 26 abr. 2012.

Oficina de qualificação do NASF. Secretaria de Atenção à Saúde. Departamento de Atenção Básica. 2010c. Disponível em: $\quad$ <http://bvsms.saude.gov.br/bvs/publicacoes/oficina_ qualificacao_nasf.pdf>. Acesso em: 27 abr. 2012.

Ministério da Saúde. Portaria no 154, de 24 de janeiro de 2008. Cria os Núcleos de Apoio à Saúde da Família - NASF. Diário Oficial da República Federativa do Brasil. Brasília-DF, 25 jan. 2008. Disponível em: <http://bvsms.saude.gov.br/bvs/saudelegis/ gm/2008/prt0154_24_01_2008.html >. Acesso em: 15 abr. 2012.

CAMPOS, G.S. Apoio matricial e equipe de referência: uma metodologia para gestão do trabalho interdisciplinar em saúde. Cadernos de Saúde Pública. Disponível em: <http://www.scielosp. org/pdf/csp/v23n2/16.pdf>. Acesso em: 22 abr. 2012.
CAMPOS, G.W.S.; DOMITTI, A.C. Apoio matricial e equipe de referência: uma metodologia para gestão do trabalho interdisciplinar em saúde. Cadernos de Saúde Pública. Disponível em: <http:// www.scielosp.org/scielo.php?script=sci_arttext\&pid=S0102311X 2007000200016\&lang=pt\&tlng $>$. Acesso em: 28 abr. 2012.

FIGUEIREDO, E.N. A Estratégia Saúde da Família na Atenção Básica do SUS. UNIFESP. UMA-SUS. 2012. Disponível em: <http://www. unasus.unifesp.br/biblioteca_virtual/esf/2/unidades_conteudos/ unidade05/unidade05.pdf>. Acesso em: 30 jan. 2013.

MÂNGIA, E.F.; LANCMAN, S. Núcleos de Apoio à Saúde da Família: integralidade e trabalho em equipe multiprofissional. Revista de Terapia Ocupacional da Universidade de São Paulo; São Paulo, v.19, n.2, ago. 2008.

MARTINES, W.R.V.; CHAVES, E.C. Vulnerabilidade e sofrimento no trabalho do Agente Comunitário de Saúde no Programa Saúde da Família. Revista da Escola de Enfermagem da USP, v.41, n.3, p.426433. 2007.

MENDONÇA, C.S. Saúde da Família, agora mais do que nunca. Ciências e Saúde Coletiva. Disponível em: <http://www.scielosp. org/scielo.php?pid=S141381232009000800022\&script=sci_ arttext>. Acesso em: 25 abr. 2012.

NASCIMENTO, D.D.G.; OLIVEIRA, C.M.A. Reflexões sobre as competências profissionais para o processo de trabalho nos Núcleos de Apoio à Saúde da Família. O Mundo da Saúde, São Paulo, v. 34, n. 1, p. 92-96. 2010.

Recebido para publicação em outubro de 2012

Versão final em outubro de 2013

Conflito de interesse: não houve

Suporte financeiro: inexistente 